cannot be extended to any surfaces of variable curvature. (Received January 30, 1945.)

69. Edward Kasner and John DeCicco: Some properties of minimal surfaces.

It is known that for any minimal surface and also for a sphere, any parallel system of plane sections is an isothermal family on the surface. The authors prove that this property is characteristic for the minimal surfaces and the spherical sur faces. Moreover the following fundamental theorem is obtained. If a surface possesses more than four distinct isothermal systems of parallel plane sections, all of which are parallel to a given rectilinear direction $L$, then every parallel system of plane sections is isothermal, and the surface is either minimal or spherical. Some further results are the following. Any surface which has more than two simply-infinite families of plane geodesics of nonzero curvature is either a sphere or a plane. If a surface has a single isothermal system of parallel plane suections such that the mean curvature is the same at all points of any one of these sections, then it is a surface of revolution. (See for related theorems paper in Proc. Nat. Acad. Sci. U.S.A. vol. 31 (1945) (Received January 24,1945 .)

\title{
Statistics and Probability
}

\section{E. J. Gumbel: Expected values of a statistical variate.}

Let $x$ be a continuous unlimited variate; let $F(x)$ be the probability of a value equal to, or less than, $x$. What values may be expected in a sample of size $n$ ? The $n$ expected values $\bar{x}_{m}(m=1,2, \cdots, n)$ and the $n$ most probable values $\tilde{x}_{m}$ are, respectively, the means and the modes of the distributions of the $m$ th values. As the calculation of these means is in general extremely tiresome, and the calculation of the $n$ modes laborious, it is proposed to use as approximations the values $\dot{x}_{m}$ which correspond to the probabilities $F_{m}$ obtained from linear interpolation between $F_{1}$ and $F_{n}$. Here, $F_{1}$ and $F_{n}$ are the probabilities of the most probable largest and of the most probable smallest value in a sample of size $n$. The probabilities $F_{m}$ are subject to the inequalities $(m-1) / n<F_{m}<m / n$. The distribution of the values $x_{m}$ converges, with increasing sample size, toward the theoretical distribution. For a symmetrical distribution the mean of the values $\hat{x}_{m}$ coincides with the mean of the population. (Received January 22, 1945.)

\section{TOPOLOGY}

71. R. H. Sorgenfrey: $A$ condition that a continuum be irreducible between two points.

It is proved that in order that a (compact) continuum $M$ be irreducible between two of its points, it is necessary and sufficient that of every three continua whose sum is $M$, either two fail to intersect or the sum of two contains the third. (Received December 4, 1944.)

\section{P. A. White: On new types of regular convergence.}

New types of convergence are introduced making use of other local properties in the same way that local connectedness was used in the definition of $r$-regular con- 